\title{
Efficient Energy Routing Protocol based on Energy $\&$ Buffer Residual Status (EBRS) for Wireless Sensor Networks
}

\begin{abstract}
Amairullah Khan Lodhi, M
orks consist of nodes, having the
Abstract- Wireless networks consist of nodes, having the
ability that, they can sense and collect the information from the ability that, they can sense and collect the information from the
nearby surroundings. It has the responsibility of designed protocol to send this collected information by data gathering and forward it to the outside network via a sink node. Furthermore, WSNs doesn't need any predetermined network structure; all the nodes used in WSN can operate as a router as well as the host. It uses multiple hops to send information to the node outside the communication range through different neighbor nodes. All the sensor nodes in WSN have their range of communication and can send and collect messages straight to each other until they were in the communication range. Moreover, the Self-organizing property of nodes in the network made WSN outstanding amongst the major applications. Nevertheless, the wireless nodes there in the network have a battery with restricted energy and can't be recharge or change once deployed. Hence, the node energy must be utilized efficiently for various functions as sensing the information, processing the sensed information, and transmitting the processed information to another node. With the enhancements of the innovation and cost-effective hardware, our visualization presents a tremendous life enhancement of WSN into several new applications. To modify following such background, the energy-efficient routing protocol is extremely desirable and can be achieved by clustering in WSN. In the literature survey, various energy-efficient routing techniques based on cluster have been given to attain the energy-efficiency and enhance the lifetime of the network. However, these protocols were suffering from the bottleneck node issue. It is the situation in the network where the router node subjected to heavy traffic due to its presence in energy-efficient routing path or high remaining energy. This paper aims to moderate the possibility of the node to become a bottleneck node throughout the application. Thus, we attain the objective by design and develop the cluster-based efficient-routing protocol by selecting the head nodes of the cluster based on their residual energy and buffer status. Performance outcome shows that the projected work out-performs in contrast with present cluster-based routing protocols.
\end{abstract}

Keyword: - Wireless Sensor Network; Sink Node, Cluster Head, Energy efficiency, Buffer, Routing, Network Lifetime, Mobile Sink Node, and Control Packets.

\section{INTRODUCTION}

The wireless sensor networks (WSNs) are built up of "nodes" commencing a small amount to numerous hundreds or sometimes thousands. Moreover, all nodes are associated with one or numerous sensor nodes.

\section{Revised Manuscript Received on December 15, 2019}

Amairullah Khan Lodhi, Ph.D. Scholar, ECE Department, Vignan's Foundation for Science, Technology \& Research ( Deemed to be University), Vadlamudi, Guntur, India. lak resumes@yahoo.co.in

M.S.S RUKMINI, ECE Department, Vignan's Foundation for Science, Technology \& Research ( Deemed to be University), Vadlamudi, Guntur, India mssrukmini@gmail.com

Dr. Syed Abdulsattar, Principal \& Professor, ECE Dept Nawab Shah Alam Khan College of Engineering \& Technology, New Malakpet, Hyderabad, India syedabdulsattar1965@gmail.com
These wireless nodes have different parts: like an energy source, an electronic circuit for interfacing, a microcontroller, a radio transceiver, and usually a battery or nowadays a power harvesting module in the embedded form [1].

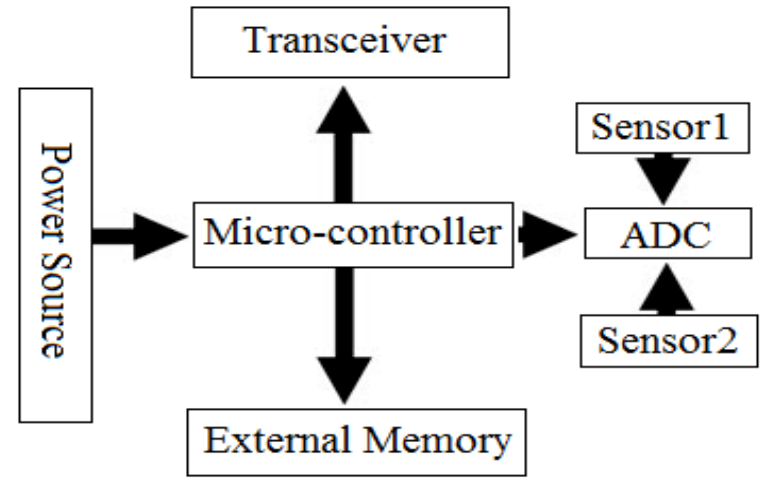

Fig. 1: Basic Structure of Sensors

The three main functions performed by all the wireless nodes are sensing the nature, preprocessing \& storage of data information with transmission along with the nodes and with the destination (sink). The WSNs is an isolated system with different sensor nodes to gather and forward the data from surrounding sensor nodes or environment after processing them [2]. The figure below shows various SNs which collects the information from the nearby atmosphere and transfer the collected information to the target node (sink) through gateway [3].

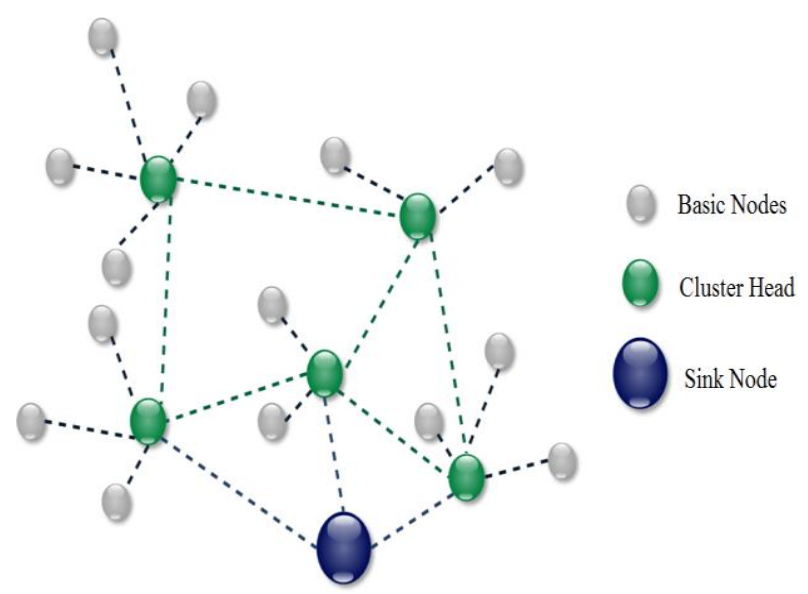

Fig.2. the basic structure of WSN

Characteristics of a good wireless network includes the minimization in consumption of energy for nodes with battery as a source, scalability to a large scale of distribution, node failure handling capacity

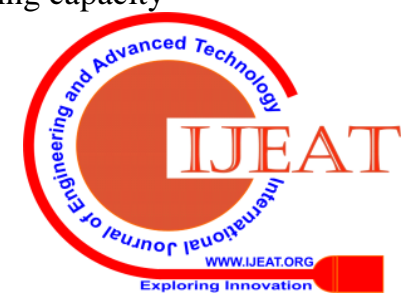


(responsiveness), simple in usage, designed in crosslayered, should have the capability to ensure severe surrounding conditions, reliability and mobility [4]. In several applications, energy-efficient data forwarding is supposed to be a critical challenge, due to inadequate energy sources \& constrained and low-powered battery issues [5]. Hence, it is insisted that the consumption of energy should be managed (Minimized) so that the network lifetime will be enhanced significantly. Routing in any network is nothing but selecting an efficient (Energy) path to process and transport the data in the network, or over several networks. This action normally maintains information of the path to several network base and forwarding based on the routing tables [6]. Furthermore, the consumption of energy and the lifetime of the network will be affected by the reduced packet delivery ratio. Especially in the case of WSN, the routing mechanism should be handled carefully because it is a very important task [7].

The residual part of the work is prearranged as the $\mathrm{II}^{\text {nd }}$ part will talk about the survey of several routing techniques and their related protocols. Section III $^{\text {rd }}$ gives our projected workout which explains the new metric for different calculation outputs with an algorithm in WSNs. Moreover, Section IV $^{\text {th }}$ deals with performance analysis of the projected routing protocol. As a final point, the conclusion is made in the $\mathrm{V}^{\text {th }}$ section.

\section{LITERATURE SURVEY}

Most of the traditional systems are more expensive as well as inappropriate for limited-resourced wireless networks. Therefore, it is extremely difficult to design an efficient energy WSN, because the wireless nodes have very low computational time, as well as less storage capacity to store data information packets [8]. Modern technological advancements in wireless technology have been approved to fabricate minimum cost, tiny, battery-operated multifunctional sensor nodes. For gathering or dissemination of data, each wireless node communicates with other nearby nodes as well as with the base station node [9]. Hence, different efficient-energy, as well as balanced- energy protocols for routing technique have been proposed for WSNs. These protocols try to minimize the consumption of energy in each $\mathrm{SN}$, in account; increases the complete network lifetime.

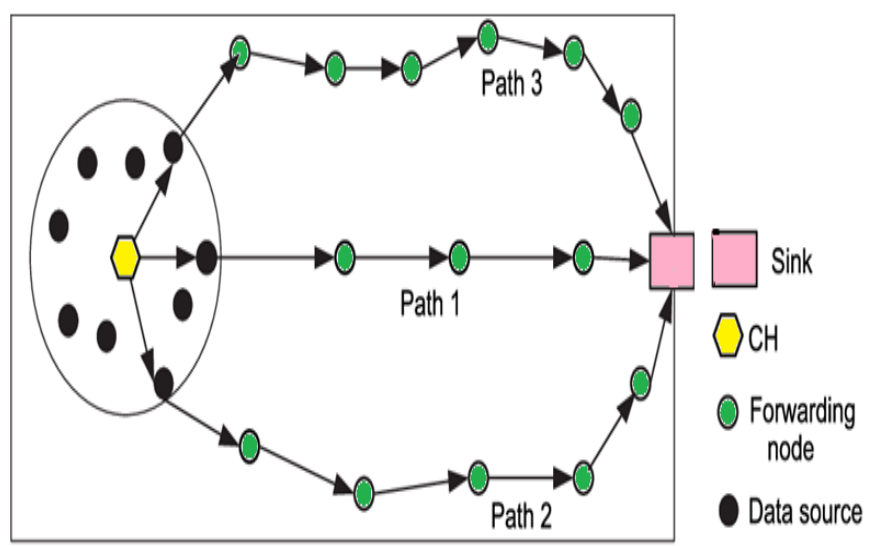

Fig.5 Different paths in WSN

The term "network lifetime" is a basic assessment metric for the particular network. It can be defined as the utmost time in which the system is efficient to measure a physical significance or occurrence. After every network data transmission around, the measurement of the nodes lifetime is done by its level of remaining energy. This remaining node energy is referred to as the residual energy of the wireless node [10].

In the earlier period, remote locations and restricted or hazardous areas, rotating machinery, and mobile resources are unreachable but now are reaching with the help of $\mathrm{I} / \mathrm{O}$ devices in wireless networks. In water industries, these $\mathrm{I} / \mathrm{O}$ devices and sensors can be powered by solar panels or battery packs [11]. The transmission capability of the node is restricted due to its communication range and hence the information data is transmitted several times through transitional nodes (Hop) via which fallout in flooding of packets which can cause the conflict between the packets, information idleness, and energy dissipation [12]. On the other hand, the queuing and delay issues are mainly universal features for the technical surroundings, such as in networking and telecommunications [13]. "Queuing premise provides the assessor with a powerful implement for designing and evaluating the performance of the queuing systems".

Except for the routing protocols, some additional techniques are there which are helpful in the transmission of data such as packet scheduling. A typical scheduler model is shown in Fig. below, in which the data packet is placed into the well-organized queue and waiting for being processed when received by the wireless node. In-network patterns like "many-to-one" and "mostly-off", all wireless nodes through the multi-hop transmissions technique generate and transmit the packets to the base node or sink.

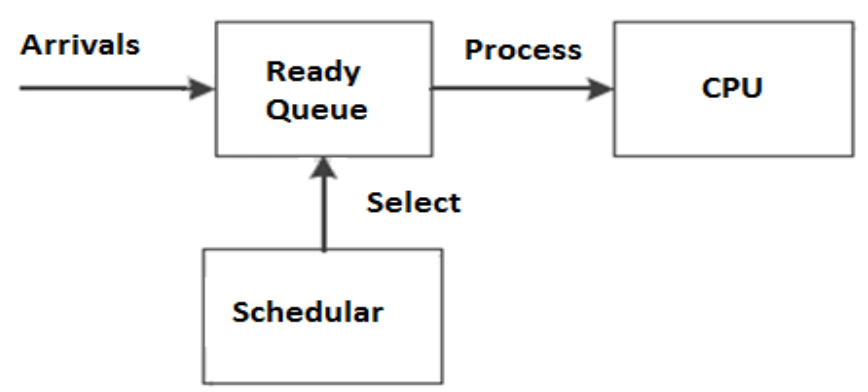

Fig.6. Basic Scheduling Scheme for maintaining queue in WSN

For that purpose, the goal is to make the wireless nodes to deplete their energy approximately at the same time, which can be done by tackling the problem on both levels i.e. on the region and at node level [14]. Hence, in the queuing model, the sensor node is blocked when the waiting queue length reaches the maximum value [15].

Some of the important metrics which are very useful for getting energy efficiency with NL elaboration are Packet delivery performance, which indicates the lifetime of the network. Throughput talks about the successful delivery of the packet to the destination per unit of time over a particular channel. The energy-efficiency metric helps in preserving the energy of the node and is considered as a sensitive parameter and for making it efficient the nodes when they are not in use for long are set to a sleep mode [16]. 


\section{PROJECTED WORKOUT}

It is mentioned in three sections

1. Cluster Head $(\mathrm{CH})$ Selection based on nodes present energy \& buffer rank

2. Detection of bottleneck Cluster Head $(\mathrm{CH})$ Node by the systematic Approach Model

3. Routing based on $\mathrm{CH}$ rank of the Mobile sink

\section{Cluster Head (CH) Selection based on nodes present energy \& Buffer rank}

As all the sensing information of the network is forwarded through the $\mathrm{CH}$ to sink node, hence $\mathrm{CH}$ selection in WSN is a considerable work. If elected $\mathrm{CH}$ is unable to hold the traffic in that case involuntarily the packets drop from it either due to lack of nodes buffer overflow or energy. Thus $\mathrm{CH}$ selection should be done precisely, as every node in a network having limited energy and buffer capacity and recharging or replacing the battery during the mission is not possible. For that purpose, a mechanism is developed for selecting the $\mathrm{CH}$ based on the status of energy as well as buffer status. It is named as "Energy \& Buffer Residual Status (EBRS)", which will help to control the packet drop from $\mathrm{CH}$ due to buffer overflow or lack of energy. Thus the projected metric extends the network performance by reducing the packet drop, which justified as follows;

The assumptions made that, every node have the energy of ' $E$ ' joules and can forward ' $N$ ' number of packets with buffer ' $B$ ' and can store ' $n$ ' number of packets in the time interval.

Consider the packet ' $P$ ' is going to forward from one of the nodes during the ' $t$ ' interval of time, this packet consume the ' $E p$ ' energy and occupies the buffer space of ' $B p$ ' then the residual status of node in respect with buffer ' $B r$ ' and energy ' $E r$ ' can be computed by following equations.

$$
\begin{gathered}
E r=N-E p, N>E p \\
\quad B r=n-B p, n>B p .
\end{gathered}
$$

The ability of a node to hold the packet in its concerning buffer and energy is computed by the knapsack algorithm by applying the following considerations

Table 1: Alphabets used and their Abbreviation

\begin{tabular}{|l|l|}
\hline \multicolumn{1}{|c|}{ Alphabets } & \multicolumn{1}{c|}{ Description } \\
\hline $\mathrm{E}$ & Battery Energy in Joules \\
\hline $\mathrm{N}$ & Number of Packets forwarding \\
\hline $\mathrm{PE}$ & Node Packets \\
\hline $\mathrm{P}_{\mathrm{B}}$ & Buffer Packets \\
\hline $\mathrm{T}$ & Period \\
\hline $\mathrm{E}_{\mathrm{r}}$ & Node Residual energy \\
\hline $\mathrm{B}_{\mathrm{r}}$ & Node Residual Buffer \\
\hline $\mathrm{Pei}$ & $\begin{array}{l}\text { The individual energy used for node } \\
\text { packet }\end{array}$ \\
\hline $\mathrm{Pbi}_{\mathrm{n}}$ & $\begin{array}{l}\text { The individual buffer used for node } \\
\text { packet }\end{array}$ \\
\hline $\mathrm{ORmax}$ & The upper limit threshold value \\
\hline $\mathrm{ORmin}$ & Lowest threshold value \\
\hline $\mathrm{B}$ & Total Available Buffer space of a node \\
\hline $\mathrm{P}_{\mathrm{m}}$ & Total Available Number of packets \\
\hline
\end{tabular}

1. In available energy and buffer space, the nodes are free to process the packets through node buffer

2. In respect to energy and buffer, the node should process the maximum number of packets

3. Partial processing of packets is not possible.

For processing ' $n$ ' number of packets successfully from a specified node exclusive of dropping it, the node must satisfy the below conditions, because the ability of the node to mitigate packet drop is 'Pn' number of packets.

1. The packet should be processed by a node completely

2. The packet should be dropped from the node, except incomplete progression should not happen

\section{Detection of bottleneck Cluster Head (CH)} Node by the Systematic Approach Model

To extend the network performance in respect with lifetime as well as packet delivery the buffer space of the node should be enough to process the packet, otherwise, a lot of packet forwarding chances gets wasted, and it will affect the network performance. Alternatively, at this time also if the node provides the space for upcoming packets, then it must drop any packet from its buffer. In both the situations, node cases as congestion that is a negative impact on the delivery of packets.

Existing protocols relating to WSN can be categorized based on;

1. Congestion control Routing

2. Congestion Non-control Routing

The majority of the obtainable protocols designed for WSN are congestion non-control. Unlike well-established networks, a dynamic and constrained network in WSN is very expensive in terms of overhead as well as time and is only possible to remove blockage but not prevent altogether. To eliminate network congestion, IETF recommended using the average queue size monitoring and computing process Thus, we compute the average queue and average waiting time of the packet inside the node buffer to control the congestion, and thereby packet loss. Thus this section aims to compute the average packet number queued in nodes buffer, if this queue size is above the buffer threshold size then this node is going to lose the packets.

Without the loss of generality, we can calculate the average numbers of packets arriving at a particular time interval by using the exponential weighted moving average method.

$$
\mathrm{A}=\alpha \mathrm{Ac}+\beta \text { Ap. }
$$

Similarly, calculation of the average amount of packets departing in an exact time interval by using the exponential weighted moving average method is given by,

$$
\mathrm{D}=\alpha \mathrm{Dc}+\beta \mathrm{Dp}
$$

Where, Ac and Dc is an average number of arrivals and departure rate of the packets in a current time interval. Conversely, Ap and Dp is an average number of arrival and departure rate of the packets in the previous time interval. The $\alpha$ and $\beta$ are the weighted constants, their values vary 
from $(0,1)$. If the condition $(A>D)$ occurs then the packet will drop from the node buffer, and If the condition $(A<D)$ occurs then packets will drop if the queue is greater than the buffer size.

Finally, the queue size is computed by

$\mathrm{Np}=(\mathrm{A}(\mathrm{D}-\mathrm{A}) /)$

Queue size $(\mathrm{QS})=\mathrm{Qp} *$ Packet size.

Furthermore, if the size of the queue is greater than the size of the buffer (threshold value i.e. buffer size/4), then the node becomes a bottleneck and it will start dropping the packet.

\section{Routing based on $\mathrm{CH}$ rank of Mobile sink}

The mobile node (sink) maintains the distance table of $\mathrm{CH}$ node in its memory table. The network creates a clusterbased network model with the help of existing "LEACH" protocol initially. Then, the mobile sink node moves randomly by Random Way Point Mobility (RWPM) Model to collect the location of CH's. After that those CH's computes the average distance between the $\mathrm{CH}$ 's by the following equation;

$$
D_{\text {avg }}=\frac{\sum_{i=0}^{m-1} \sqrt{\left(X_{i}-X_{c}\right)^{2}+\left(Y_{i}-Y_{C}\right)^{2}}}{m-1} i \neq c
$$

Where,

\section{$\left(X_{c}, Y_{C}\right)=$ Location of the current cluster head \\ $\left(X_{i}, Y_{i}\right)=$ Location of the cluster head \\ $m=$ Total number of cluster heads}

After a particular time interval or whenever a new $\mathrm{CH}$ is got elected, the memory table of the Mobile Sink Node (MSN) is updated. The MSN selection of the $\mathrm{CH}$ is done by two factors, Energy and Buffer (E\&B), which is calculated in the previous section and moves it towards the communication process. The Control Packets (CP) is used to inform the $\mathrm{CHs}$, that the MSN is visited it. Every $\mathrm{CH}$ in LEACH protocol is active only for a particular amount of time regarding its energy parameters. If new $\mathrm{CH}$ is elected for the cluster then MSN compares its residual status (E\&B) with remaining $\mathrm{CHs}$ residual status, if it finds any other node has more status than the current $\mathrm{CH}$ status, then MSN moves towards new $\mathrm{CH}$ and this process continues till the completion of the task. The proposed algorithm is explained as follows,

1. Based on LEACH routing protocol divide the network into different clusters and elect the $\mathrm{CH}$.

2. Now based on the previous section the $\mathrm{CHs}$ will compute their current status of energy and load.

3. The MSN, by moving nearer to $\mathrm{CHs}$, will collect the information about distance as well as $\mathrm{CHs}$ present status, and maintain it in the vector table.

4. Now according to the vector table, decide the lifetime of $\mathrm{CH}$ and assume it as a period ' $\mathrm{T}$ ' (This is the time for which sink node will collect the information from the current $\mathrm{CH}$, and create the communication sessions with the $\mathrm{CH})$.

5. The sensing information from cluster members will be collected through the $\mathrm{CH}$ and pass on it to the MSN.

6. After this session, $\mathrm{CH}$ reselection process will begin and now it is purely based on their remaining buffer and energy status. If $\mathrm{CH}$ is selected, then the MSN will compute the distance as well as status and updates the vector table and compare the value with other $\mathrm{CH}$ values. If it found greater, then MSN will set the communication session time interval and start communication. Otherwise, MSN will move towards the $\mathrm{CH}$ which has higher status regarding energy \& buffer and start communication.

7. This process will continue until the end.

\section{PERFORMANCE ANALYSIS}

Performance assessment of projected system is done by NS-2 (NS-2.34) with suitable expansion inaccessible libraries and compare through present protocols in the same network environment. We assumed the communication space of each node is $250 \mathrm{~m}$ and single-hop broadcast delay is $10 \mathrm{~ms}$, in our model.

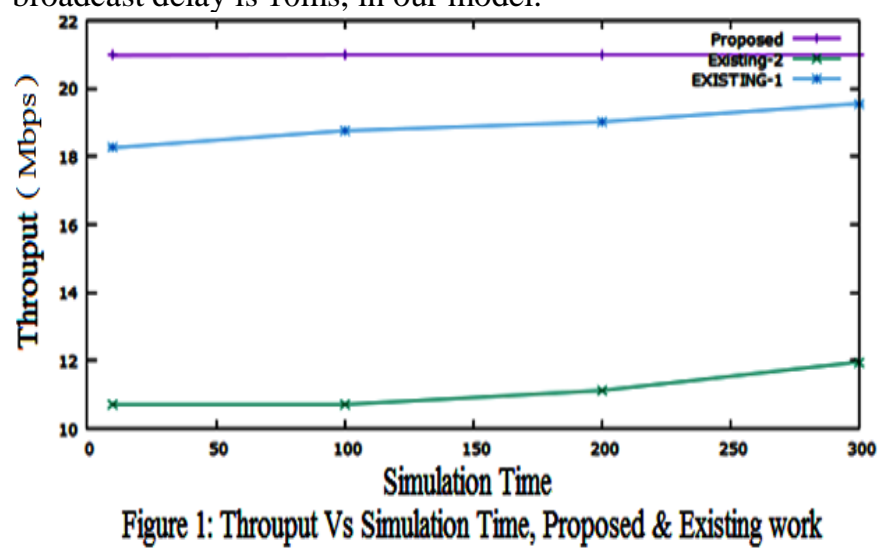

All wireless nodes are set crosswise $1000 \times 1000$ square area. Nodes are outfitted through $10 \mathrm{j}$ battery, data packets are of size 512 bytes with the power consumption of $600 \mathrm{~mW}$ for transmission, and $300 \mathrm{~mW}$ is receiving the power of the node. Every node must run our cluster head selection system and the cluster head transmit its beacons more than two hops every $20 \mathrm{sec}$.

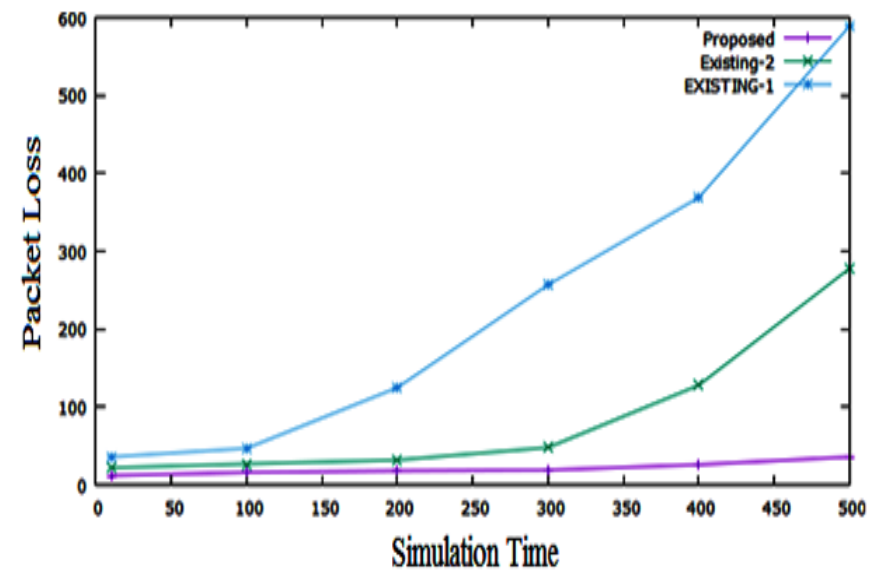

Figure 2:- Packet Loss Vs Simulation Time, Proposed \& Existing Work

For that reason, we evaluate the network performance concerning the loss of packet and network lifetime and evaluate it with the present protocols.

Published By: 


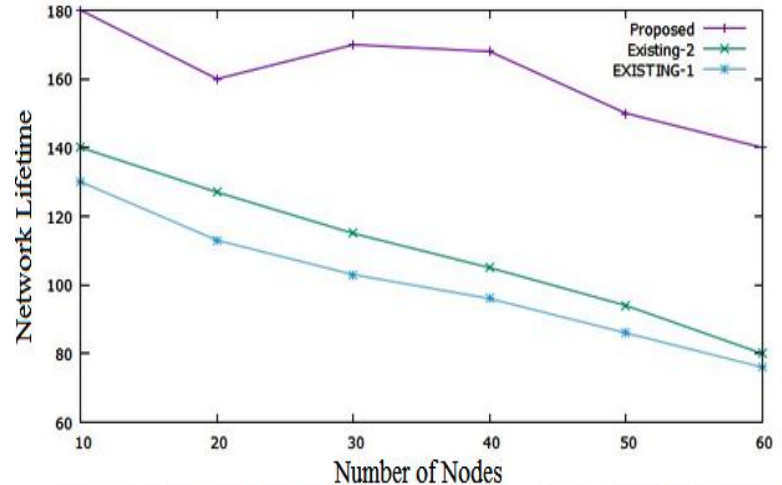

Figure 3:- Network Lifetime Vs Number of Nodes, Proposed \& Existing Work

Figures 1, 2 and 3 noticeably show that projected system improve the system performance and elaborate the lifetime of the network.

\section{CONCLUSION}

To mitigate the bottleneck issue in WSN the suitable approach is cluster-based routing due to its less overhead in energy-efficiency and buffer. However, if merely one parameter i.e., energy efficiency, is considered for the selection of cluster heads then it may results in packet loss due to inadequate buffer. Hence, it is very essential that both parameters i.e., energy and buffer should be considered to select the cluster head in wireless networks. Furthermore, the degradation in network lifetime, depletion in network resources, and overhead are caused by the negligence of bottleneck node which is also a considerable issue to mitigate packet drop in WSNs. Hence, a novel metric "Energy \& Buffer Residual Status (EBRS)" is put in place to select the cluster head which gives the status of the wireless node regarding its residual position of the energy of the node as well as its buffer. Moreover, this proposed metric will elaborate the lifetime of the network and accumulate the node energy. Additionally, it also monitors the current status of the wireless nodes and hence may be used like proactive routing protocol for cluster head selection which is our upcoming task.

\section{REFERENCES}

1. Mahgoub, Imad, and Mohammad Ilyas. Sensor network protocols. CRC Press, 2018.

2. Modieginyane, Kgotlaetsile Mathews, Babedi Betty Letswamotse, Reza Malekian, and Adnan M. Abu-Mahfouz. "Software defined wireless sensor networks application opportunities for efficient network management: A survey." Computers \& Electrical Engineering 66 (2018): 274-287.

3. Cerchecci, Matteo, Francesco Luti, Alessandro Mecocci, Stefano Parrino, Giacomo Peruzzi, and Alessandro Pozzebon. "A low power IoT sensor node architecture for waste management within smart cities context." Sensors 18, no. 4 (2018): 1282.

4. Bedi, Guneet, Ganesh Kumar Venayagamoorthy, Rajendra Singh, Richard R. Brooks, and Kuang-Ching Wang. "Review of Internet of Things (IoT) in electric power and energy systems." IEEE Internet of Things Journal 5, no. 2 (2018): 847-870.

5. Rahmani, Amir M., Tuan Nguyen Gia, Behailu Negash, Arman Anzanpour, Iman Azimi, Mingzhe Jiang, and Pasi Liljeberg. "Exploiting smart e-Health gateways at the edge of healthcare Internet-of-Things: A fog computing approach." Future Generation Computer Systems 78 (2018): 641-658.

6. Casado, Martin, Keith E. Amidon, Peter J. Balland III, Natasha Gude, Justin Pettit, Benjamin L. Pfaff, Scott J. Shenker, and Daniel J. Wendlandt. "Network operating system for managing and securing networks." U.S. Patent Application 15/838,317, filed April 12, 2018.

7. Modieginyane, Kgotlaetsile Mathews, Babedi Betty Letswamotse Reza Malekian, and Adnan M. Abu-Mahfouz. "Software defined wireless sensor networks application opportunities for efficient network management: A survey." Computers \& Electrical Engineering 66 (2018): 274-287.

8. Sánchez-Álvarez, David, Marino Linaje, and Francisco-Javier Rodríguez-Pérez. "A Framework to Design the Computational Load Distribution of Wireless Sensor Networks in Power Consumption Constrained Environments." Sensors 18, no. 4 (2018): 954.

9. Garcia-Luna-Aceves, Jose J. "System and method for efficient name-based content routing using link-state information in information-centric networks." U.S. Patent Application 10/003,520, filed June 19, 2018

10. Sundararaj, Vinu, Selvi Muthukumar, and R. S. Kumar. "An optimal cluster formation based energy efficient dynamic scheduling hybrid MAC protocol for heavy traffic load in wireless sensor networks." Computers \& Security 77 (2018): 277-288.

11. Bhargavi, Peyakunta, and Singaraju Jyothi. "Big Data and Internet of Things for Analysing and Designing Systems Based on Hyperspectral Images." In Environmental Information Systems: Concepts, Methodologies, Tools, and Applications, pp. 621-641. IGI Global, 2019.

12. Aalsalem, Mohammed Y., Wazir Zada Khan, Wajeb Gharibi, Muhammad Khurram Khan, and Quratulain Arshad. "Wireless Sensor Networks in oil and gas industry: Recent advances, taxonomy, requirements, and open challenges." Journal of Network and Computer Applications 113 (2018): 87-97.

13. Iqbal, Rahat, Faiyaz Doctor, Brian More, Shahid Mahmud, and Usman Yousuf. "Big data analytics: Computational intelligence techniques and application areas." Technological Forecasting and Social Change (2018).

14. Ezhilarasi, M., and V. Krishnaveni. "A survey on wireless sensor network: energy and lifetime perspective." Taga Journal of Graphic Technology 14 (2018).

15. Zhang, Zeyu, Amjad Mehmood, Lei Shu, Zhiqiang Huo, Yu Zhang, and Mithun Mukherjee. "A survey on fault diagnosis in wireless sensor networks." IEEE Access 6 (2018): 11349-11364.

16. Wang, Xu, Xuan Zha, Wei Ni, Ren Ping Liu, Y. Jay Guo, Xinxin Niu, and Kangfeng Zheng. "Survey on blockchain for Internet of Things." Computer Communications (2019). 